\title{
Theoretical study of stability and charge-transport properties of coronene molecule and some of its halogenated derivatives: A path to ambipolar organic-based materials?
}

\author{
J. C. Sancho-García ${ }^{a)}$ and A. J. Pérez-Jiménez ${ }^{\text {b) }}$ \\ Departamento de Química Física, Universidad de Alicante, E-03080 Alicante, Spain
}

(Received 22 July 2014; accepted 23 September 2014; published online 7 October 2014)

\begin{abstract}
We have carefully investigated the structural and electronic properties of coronene and some of its fluorinated and chlorinated derivatives, including full periphery substitution, as well as the preferred orientation of the non-covalent dimer structures subsequently formed. We have paid particular attention to a set of methodological details, to first obtain single-molecule magnitudes as accurately as possible, including next the use of modern dispersion-corrected methods to tackle the corresponding non-covalently bound dimers. Generally speaking, this class of compounds is expected to self-assembly in neighboring $\pi$-stacks with dimer stabilization energies ranging from -20 to $-30 \mathrm{kcal} \mathrm{mol}^{-1}$ at close distances around 3.0-3.3 $\AA$. Then, in a further step, we have also calculated hole and electron transfer rates of some suitable candidates for ambipolar materials, and corresponding charge mobility values, which are known to critically depend on the supramolecular organization of the samples. For coronene and per-fluorinated coronene, we have found high values for their hopping rates, although slightly smaller for the latter due to an increase (decrease) of the reorganization energies (electronic couplings). () 2014 AIP Publishing LLC. [http://dx.doi.org/10.1063/1.4897205]
\end{abstract}

\section{INTRODUCTION}

The flourishing field of organic electronics is maturing so rapidly that we can already observe multiple initiatives from academic laboratories and industrial partners to develop new technologies and devices, in an attempt to revolutionise the whole society and create a robust platform of economic and sustainable growth. The word organic refers to the use of organic materials, be them polymers, oligomers, or small molecules, as active compounds for these device applications, contrarily to currently produced inorganic-based devices. The large envisioned applications of the former range from electronic and optical devices, consumer electronics and screens, advanced batteries, medical applications and aids, clean energy devices, sensors and membranes, and light-harvesting antennas, among others. The efficiency of a wide part of these devices is dominated by the charge carriers mobility, which characterises how quick a particle carrying a charge will move across the active layer upon application of an external electric field. ${ }^{1,2}$ This magnitude is normally obtained in theoretical calculations after estimating the corresponding charge transfer rates, which also means to know in advance the possible pathways to be followed by particle carriers depending on the obtained solid-state (sometimes necessarily idealised) morphologies.

Upon doping from external sources, a successful organicbased candidate for electronic devices should be able to transport charge carriers of both existing types, electrons and holes, behaving thus respectively as an ambipolar $n$ type and $p$-type conductor. ${ }^{3}$ Many known organic materi-

\footnotetext{
a)E-mail: jc.sancho@ua.es

b)E-mail: aj.perez@ua.es
}

als show excellent $p$-type performance, mainly elucidated due to the intense research performed in last years, with the selection of materials for $n$-type behavior being now under closer scrutiny. ${ }^{4-6}$ The path followed for achieving $n$-type organic semiconductors is based on the large body of knowledge existing for corresponding $p$-type systems. For instance, functionalization of the molecule with active electron-withdrawing groups is a recurrent strategy, be these groups cyano, imide, or halogen atoms, as well as heteroatom replacements of the carbon atoms within the molecular backbone. ${ }^{7-10}$ For instance, the pentacene molecule, taken this case as an illustrative example, is considered a prototype $p$-type material, ${ }^{11}$ whereas its per-fluorination allowed to obtain electron mobilities as high as $0.11 \mathrm{~cm}^{2} \mathrm{~V}^{-1} \mathrm{~s}^{-1}$ compared to hole mobilities of $0.45 \mathrm{~cm}^{2} \mathrm{~V}^{-1} \mathrm{~s}^{-1}$ under the same experimental conditions, showing thus comparable mobilities and ambipolar behavior. ${ }^{12}$ Another strategy currently pursued in hydrocarbons as $2,6^{\prime}: 2^{\prime}, 6^{\prime \prime}$-terazulene revealed an asymmetrical distribution of the frontier molecular orbitals leading to (in this case) a preferred $n$-type behavior. ${ }^{13}$

In an attempt to disclosure existing relationships between specific substitution patterns and corresponding ambipolar (if any) behavior, we have chosen to start from a 2D-shaped molecule such as coronene (hexabenzobenzene), see Figure 1, which has been thoroughly studied in last years, ${ }^{14-16}$ and systematically study the influence of full and partial halogenation (fluorination and chlorination) in the bulk of properties related with charge transfer. We remark that molecular order at the nanoscale is revealed of utmost importance. ${ }^{14,17}$ Hexabenzocoronene is known to form discotic liquid-crystalline materials with molecules uniaxially oriented along the transport direction with field-effect mobilities reaching $0.01 \mathrm{~cm}^{2} \mathrm{~V}^{-1} \mathrm{~s}^{-1} \cdot{ }^{18,19}$ However, the 

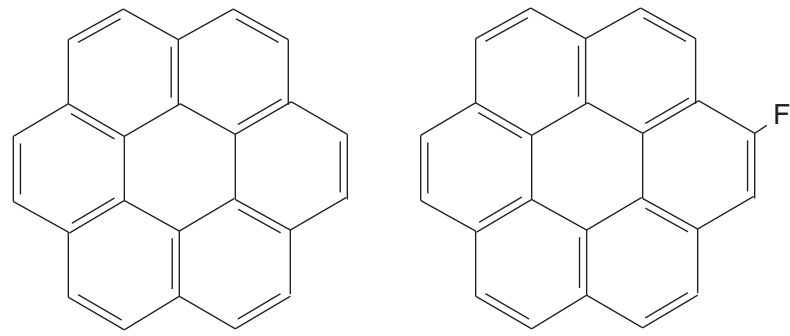<smiles>Clc1cc2ccc3ccc4ccc5ccc6ccc1c1c6c5c4c3c21</smiles><smiles>Fc1c(F)c2c(F)c(F)c3c(F)c(F)c4c(F)c(F)c5c(F)c(F)c6c(F)c(F)c1c1c2c3c4c5c61</smiles><smiles>Clc1c(Cl)c2c(Cl)c(Cl)c3c(Cl)c(Cl)c4c(Cl)c(Cl)c5c(Cl)c(Cl)c6c(Cl)c(Cl)c1c1c2c3c4c5c61</smiles>

FIG. 1. Chemical structures of coronene (unsubstituted) and its mono- and per-substituted studied molecules. The hydrogen atoms and corresponding C-H bonds have been omitted for clarity.

substitution pattern followed here may also bring additional non-covalent through-space interactions, ${ }^{20,21}$ complementarily to $\pi \cdots \pi$ and $\mathrm{C}-\mathrm{H} \cdots \pi$ interactions largely found in acene-based materials, which also deserve to be closely investigated by theoretical methods. Relying again on existing pentacene data, it is known how per-halogenation ${ }^{22}$ tune from $p$-type towards $n$-type semiconductors concomitantly with opening of the herringbone angle ${ }^{23}$ typical of acenelike structures. ${ }^{24}$ Also note that coronene-derived graphene transistors have also been successfully prepared, ${ }^{25}$ and that per-chlorocoronene has been already synthesised ${ }^{26}$ as well as some fluoroaromatic solids, acting as lubricants, composed of small fluorocoronene rings. ${ }^{27}$ However, this kind of substitution might affect many properties of the materials, ranging from enhanced stability upon moisture exposure to dramatic changes in solid-state morphology of the grown samples, which would need to be carefully investigated under the same technical conditions. This study can be thus considered as an attempt to shed light on these issues and to pave the way towards the understanding on the underlying structureproperty relationships.

\section{THEORETICAL FRAMEWORK}

\section{A. Marcus transfer rates}

We will consider in the following a weak-coupling regime, where charge transfer process is assisted by thermally activated intra- and intermolecular vibrations near roomtemperature. ${ }^{28}$ Thus, a sequence of hole/electron $\left(\mathrm{h}^{+} / \mathrm{e}^{\cdot-}\right)$ hops between neighboring molecules ( $\mathrm{M}$ and $\mathrm{N}$ ) occupying well-defined sites on a crystalline lattice

$$
\mathrm{M}^{+(-)}+\mathrm{N} \stackrel{\mathrm{k}_{\mathrm{CT}}}{\longrightarrow} \mathrm{M}+\mathrm{N}^{\cdot+(-)}
$$

will provide the driving force for the migration of charges across the active layer made of organics. ${ }^{29-32}$ Note that the reactants (products) are considered to be the initial (i) and final (f) states, respectively. Under these conditions, molecules are expected to experience large geometry relaxation upon arrival of the charge, thus leading to charge localization and selftrapping, the rate of charge transfer can be expressed as ${ }^{33,34}$

$$
\mathrm{k}_{\mathrm{CT}}=\frac{2 \pi}{\hbar}\left|\mathrm{V}_{\mathrm{if}}\right|^{2} \frac{1}{\sqrt{4 \pi \Lambda k_{\mathrm{B}} T}} \exp \left\{-\frac{\left(\Delta G^{\ominus}+\Lambda\right)^{2}}{4 \Lambda k_{\mathrm{B}} T}\right\},
$$

where $\hbar$ and $k_{B}$ are fundamental constants, and $T$ is the temperature chosen here to be $298.15 \mathrm{~K}$. Therefore, the two key energy magnitudes entering into the former equation, $\Lambda$ and $\mathrm{V}_{\text {if }}$, are known as (mainly intramolecular) reorganization energy and (intermolecular) electronic coupling, respectively, and can be accurately extracted from first-principles calculations (vide infra). Also note that $\Delta G^{\ominus}$ is the energy difference (driving force) between initial and final states, which vanishes for equivalent molecules (self-exchange reaction). Nuclear tunnelling effects, as well as disorder effects caused by vibrational fluctuations of molecules at room temperature, ${ }^{35,36}$ will not be considered; the effective incorporation of these effects would need the consideration of more complex physical models ${ }^{37-40}$ which are however beyond the scope of this study.

\section{B. Computational details}

All the calculations, unless otherwise noticed, were done here with the ORCA quantum-chemical package. ${ }^{41}$ The numerical thresholds for quadrature grids or convergence issues were always increased with respect to the defaults; the "Chain-of-Spheres" (COSX) technique ${ }^{42}$ was employed when needed to reduce the computational cost. The latter 
algorithms require the use of some auxiliary basis sets, which are however always taken from the corresponding hardwired library. ${ }^{43}$ Complementarily, the electronic coupling was calculated by the electron transfer module of NWChem $6.3,{ }^{44}$ which utilises the method of Corresponding Orbital Transformation $^{45}$ to calculate rigorously the coupling from the wavefunctions of the reactant and product states. ${ }^{46-48}$

\section{Single-molecule magnitudes}

The results from calculations of energy magnitudes related to charge transport are known to be highly dependent on the underlying theoretical details, ${ }^{49,50}$ which largely reflects the composition of the expression chosen to represent exchange-correlation effects at a nanoscopic level. One of these magnitudes is the so-called reorganization energy $(\Lambda)$, which can be viewed as the energy needed to switch from the initial $\left|\Psi_{\mathrm{M}^{+(-)}} \Psi_{\mathrm{N}}\right\rangle$ to the final $\left|\Psi_{\mathrm{M}} \Psi_{\mathrm{N}^{+(-)}}\right\rangle$molecular state, as a consequence of charge migration across the sample. This energy is normally calculated by means of the following expression: ${ }^{51}$

$$
\Lambda_{\mathrm{h}(\mathrm{e})^{+(-)}}=\left[E_{\mathrm{M}^{+(-)} / / \mathrm{N}}-E_{\mathrm{M}^{+(-)}}\right]+\left[E_{\mathrm{N} / / \mathrm{M}^{+(-)}}-E_{\mathrm{N}}\right],
$$

where $E_{\mathrm{N}}$ or $E_{\mathrm{M}^{+(-)}}$refers to total energy of the neutral or charged molecules, at their respective optimized geometries. Since charge transfer is modelled through concerted steps, then $E_{{\mathrm{N} / / \mathrm{M}^{+(-)}}}$or $E_{\mathrm{M}^{+(-)} / / \mathrm{N}}$ are the energies of the neutral or charged molecules, but at the optimized geometry of the other involved state. Also note that closed- or open-shell calculations are done for neutral or doublet states, respectively. From the set of calculations done, one can also obtain by the $\triangle$ SCF method, employing thus total energies, the corresponding Vertical/Adiabatic Ionization Potentials (VIP/AIP) as well as corresponding Electron Affinities (VEA/AEA).

Actually, when dealing with charge transport issues within the common Density Functional Theory (DFT) framework, the degree of charge localization is correctly addressed after finding a key compromise between EXact-Exchange $(\mathrm{EXX})$ energy, $E_{x}^{\mathrm{EXX}}$, in the sense of being the exact expression for exchange but calculated with the orbitals arising during these calculations, which is known to overlocalise the electronic density, and a proper exchange density functional, $E_{x}[\rho]$, which on the other hand tends to delocalise too much the electronic cloud. A balanced treatment of related systems can be achieved by fine-tuning the proportion, after defining the corresponding weight (w) between these two elements and a training set to fit it, which follows from the general-like expression

$$
E_{x c}[\rho]=\mathrm{w} E_{x}^{\mathrm{EXX}}+(1-\mathrm{w}) E_{x}[\rho]+E_{c}[\rho] .
$$

We have formerly ${ }^{52}$ optimized the value of $\mathrm{w}$ by imposing to reproduce as close as possible the experimental $\Lambda_{\mathrm{h}^{+}}$values, extracted from gas-phase UPS spectra and lying within the 100-200 meV range of values, for some oligoacenes (anthracene, tetracene, pentacene) of the most interest. ${ }^{53}$ This approach will be coined as $B_{\Lambda}$ LYP in the following, thanks to the choice of the Becke ${ }^{54}$ and Lee-Yang-Parr ${ }^{55}$ functionals to describe exchange and correlation effects in Eq. (4), respectively, finding a w value equal to 0.2533 in this case. Also note that we decided to use herein the large def2-TZVP basis set, ${ }^{56}$ to minimise as much as possible any basis sets incompleteness issue, and that this protocol $\left(\mathrm{B}_{\Lambda} \mathrm{LYP} / \mathrm{def} 2-\mathrm{TZVP}\right)$ drops an averaged Mean Absolute (Relative) Deviation of only $13 \mathrm{meV}(8.8 \%)$ for the $\Lambda_{\mathrm{h}^{+}}$values of the set composed by these anthracene, tetracene, and pentacene molecules.

\section{Calculations in dimer systems}

The stability of the tackled dimer systems was estimated in all cases by adding the dispersion energy $\left(E_{D}\right)$ to the electronic energy provided by the BLYP model. The latter is obtained by setting $\mathrm{w}=0$ in Eq. (4). The former is obtained by using the -D3 model..$^{57,58}$ (see the supplementary material. ${ }^{112}$ ) It has been shown before that inclusion of this missing energy dominates the final accuracy of results for supramolecular samples, and thus that the choice of the functional itself is of less importance, contrarily to what is expected for reorganization energies (as described above). Geometry optimizations for dimer systems are thus done at the cost-efficient BLYP-D3/def2-SVP level. The final calculations on binding energies also incorporate a further (three-body) correction ${ }^{59}$ depending on system size, ${ }^{60,61}$ and are done with a larger basis set (BLYP-D3/def2-TZVP) and can be thus considered to be nearly free of basis sets incompleteness issues.

The intermolecular electronic coupling, $\mathrm{V}_{\text {if }}$, measuring the strength of the coupling between the initial and final states, is defined ${ }^{62}$ as one half of the energy difference between the adiabatic potential energies at the geometry $\left(Q_{c}\right)$ where the diabatic (localized) potential energy surfaces cross each other,

$$
\mathrm{V}_{\text {if }}=\frac{1}{2}\left[E_{+}(Q)-E_{-}(Q)\right]_{Q=Q_{c}},
$$

and it is calculated following the way detailed in the supplementary material. ${ }^{112}$ First, we have always verified that true localized states for charge transfer reactant and product states were obtained. To do it, it was not necessary to impose any constrain in the dimer calculations to keep the charge localized on each monomer, provided that the molecular orbitals of the monomers are used as starting orbitals for the dimer, a feature made possible in NWChem by the superposition of fragment molecular orbitals. Second, the sufficiently large cc-pVDZ basis set was employed for all the calculations, although there is some evidence showing how values are relatively independent of basis sets size. ${ }^{63}$ Third, for the case of dealing with an electron transfer reaction, where the overlap between interacting molecules might need to be more accurately described within the weakly overlapping region, we will also employ the corresponding diffuse-augmented (aug-cc-pVDZ) extension as a sanity check. Last but not the least, we also note that this way (UHF) of estimating the values copes with the large variations found for electronic couplings using different density functionals, ${ }^{64}$ which are found to linearly increase in pentacene or rubrene upon larger HFlike exchange percentage contained in the hybrid exchangecorrelation functional used; ${ }^{50}$ thus, the values reported here can be considered as an upper limit with respect to experimental values. ${ }^{65}$ 


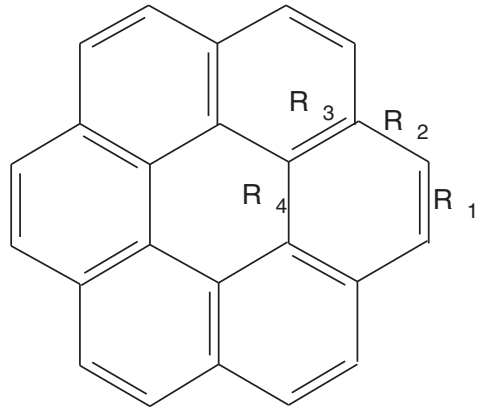

FIG. 2. Chemical structure of coronene showing some important bond lengths.

\section{RESULTS AND DISCUSSION}

\section{A. Geometry and electronic structure changes upon halogenation on single molecules}

\section{Structure and frontier orbitals}

We have first tackled mono- and per-substituted isolated coronene molecules (Figure 1) in order to study their structural and electronic properties. As a matter of reference, Figure 2 lists some important bond length values $\left(\mathrm{R}_{1}-\mathrm{R}_{4}\right)$ for the coronene case, which are (in $\AA$ ) equal to 1.366 , $1.420,1.415$, and 1.423 , respectively, at the $\mathrm{B}_{\Lambda} \mathrm{LYP} / \mathrm{def} 2-$ TZVP level. The calculated gap between the frontier orbitals amounts to $4.3 \mathrm{eV}$, with the Highest Occupied (HO) and Lowest Unoccupied (LU) Molecular Orbital (MO) energies being of the order of -5.73 and $-1.40 \mathrm{eV}$, respectively. The mono-substitution by halogen atoms does not significantly change neither the monitorized distances, as much as $0.007 \AA$, nor the energy gap, being equal now to $4.2 \mathrm{eV}$ in both cases. However, as it was expected, per-halogenation has a more marked influence on these properties, which is particularly true in the case of per-chlorination: whereas the $\mathrm{R}_{1}$ distance changes by up to $0.02 \AA$, the gap reduces to $3.5 \mathrm{eV}$, as a by-product of an unbalanced stabilization of both energy levels ( -6.50 and $-2.96 \mathrm{eV}$, for HOMO and LUMO, respectively). Interestingly, this correlates with a lower delocalisation of the electronic cloud which is mainly attributed to the degree of twisting of the backbone, as can be seen from Figure 3. This distortion from planarity was observed before even at the semi-empirical AM1 level, ${ }^{66}$ which is thus confirmed here at a much more accurate level. This re-
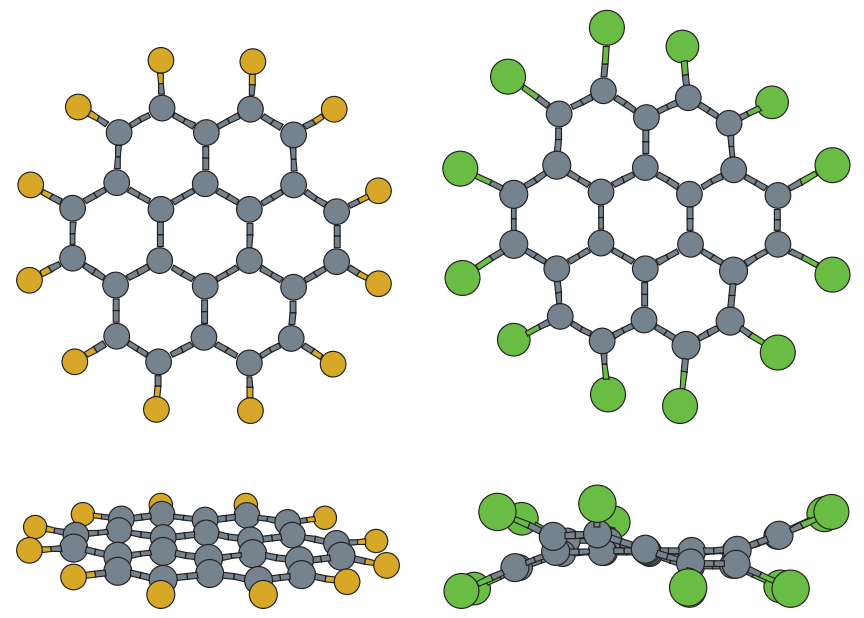

FIG. 3. Optimized structures of per-fluorocoronene (left) and perchlorocoronene (right) from perpendicular (top) and side (bottom) views.

lease of the steric hindrance due to bulky atoms was also found before in the case of partially substituted tetracene molecules (5,6,11,12-tetrachlorotetracene adopts a twisted form in agreement with the $\mathrm{X}$-ray data for this molecule ${ }^{67}$ ) or perhalogenated dibenzochrysene molecules. ${ }^{68}$ This effect is not so pronounced in the case of per-fluorination, resulting in an energy gap of $4.1 \mathrm{eV}$ coming from the $\operatorname{HOMO}(-6.77 \mathrm{eV})$ and LUMO $(-2.65 \mathrm{eV})$ energies.

\section{Ionization potentials, electron affinities, and reorganization energies}

Table I shows the evolution of first ionization potential and electron affinity of coronene upon mono- and perhalogenation, with both magnitudes increasing significantly for the latter case, by roughly up to $0.8-1.0$ and $1.2-1.8 \mathrm{eV}$, respectively; this behavior has also been observed before in the case of oligoacenes. ${ }^{69}$ Since the efficiency of charge injection, in absence of interface dipole effects, is favoured by the energy alignment between the workfunction of the metallic electrodes and the ability of organic materials to reduce or oxidize, the overall impact of these results might be a lower energy barrier for injections of electrons into per-halogenated samples. We would like to emphasise in the case of coronene that: (i) our estimates of the adiabatic ionization potential $(6.89 \mathrm{eV})$ agrees within 5\% with respect to the experimental

TABLE I. Estimates at the $\mathrm{B}_{\Lambda}$ LYP-D3/def2-TZVP level of adiabatic (A) and vertical (V) ionization potentials $(\mathrm{IP}$, in $\mathrm{eV})$ and electron affinities $(\mathrm{EA}$, in $\mathrm{eV})$, as well as intra-molecular hole $\left(\Lambda_{\mathrm{h}^{+}}\right.$, in meV) or electron $\left(\Lambda_{\mathrm{e}^{-}-}\right.$, in $\mathrm{meV})$ reorganization energies, for the coronene $\left(\mathrm{X}=\mathrm{X}^{\prime}=\mathrm{H}\right)$, mono- $\left(\mathrm{X}=\mathrm{F}\right.$ or $\left.\mathrm{Cl}, \mathrm{X}^{\prime}=\mathrm{H}\right)$, and per-substituted $\left(\mathrm{X}=\mathrm{X}^{\prime}=\mathrm{F}\right.$ or $\left.\mathrm{Cl}\right)$ studied molecules.

\begin{tabular}{lccccc}
\hline \hline & Coronene & $\begin{array}{c}\text { Mono-fluorinated } \\
\text { coronene }\end{array}$ & $\begin{array}{c}\text { Mono-chlorinated } \\
\text { coronene }\end{array}$ & $\begin{array}{c}\text { Per-fluorinated } \\
\text { coronene }\end{array}$ & $\begin{array}{c}\text { Per-chlorinated } \\
\text { coronene }\end{array}$ \\
\hline VIP (AIP) & $6.96(6.89)$ & $7.03(6.94)$ & $7.03(6.95)$ & $8.00(7.86)$ & $7.61(7.51)$ \\
VEA (AEA) & $0.19(0.29)$ & $0.38(0.43)$ & $0.37(0.48)$ & $1.43(1.57)$ & $1.96(1.79)$ \\
$\Lambda_{\mathrm{h}^{+}}$ & 144 & 172 & 162 & 283 & 221 \\
$\Lambda_{\mathrm{e}^{-}}$ & 189 & 198 & 212 & 293 & 302 \\
$\Lambda_{\mathrm{h}^{+}} / \Lambda_{\mathrm{e}^{--}}$ & 0.76 & 0.87 & 0.76 & 0.97 & 0.72 \\
\hline \hline
\end{tabular}


<smiles></smiles><smiles></smiles><smiles></smiles><smiles></smiles><smiles></smiles><smiles></smiles><smiles></smiles><smiles></smiles>

FIG. 4. Set of di-substituted coronene molecules showing the relative position of the halogen atoms $(\mathrm{X}=\mathrm{F}$ or $\mathrm{X}=\mathrm{Cl})$. The hydrogen atoms and corresponding $\mathrm{C}-\mathrm{H}$ bonds have been omitted for clarity.

value of $7.29 \pm 0.03 \mathrm{eV}$ from the photoionisation threshold $;^{70}$ (ii) our estimates of the adiabatic electron affinity $(0.29 \mathrm{eV})$ also reasonably agree with respect to available experimental values $(0.47 \pm 0.09){ }^{71}$

We now turn to the discussion of reorganization energies of these molecules upon arrival of the hole or electron particles, with the calculated values also gathered in Table I. For the coronene case, we calculate rather similar values for both $\Lambda_{\mathrm{e}^{-}}(144 \mathrm{meV})$ and $\Lambda_{\mathrm{h}^{+}}(189 \mathrm{meV})$, giving a ratio $\Lambda_{\mathrm{h}^{+}} / \Lambda_{\mathrm{e}^{-}}$equal to 0.76 and thus close to ambipolar behavior, remaining the same other features. Note also that: (i) previous estimates of reorganization energies for coronene at the B3LYP/6-311++G(d,p) level agrees within $10 \%$ or our values; ${ }^{72}$ and (ii) the use of a more sophisticated although more costly functional (the double-hybrid B $2 \pi$-PLYP model, specially devised for accurately dealing with $\pi$-conjugated systems of any type ${ }^{73}$ ) only slightly change the absolute values of $\Lambda_{\mathrm{e}^{-}}$and $\Lambda_{\mathrm{h}^{+}}$, being now of 128 and $162 \mathrm{meV}$, respectively, still keeping a similar ratio (0.79). We also observe that upon halogenation, both $\Lambda_{\mathrm{h}^{+}}$or $\Lambda_{\mathrm{e}^{-}}$increase, as it was also observed before for partially substituted tetracene molecules ${ }^{74}$ or for larger phthalocyanine systems, ${ }^{75}$ although the ratio $\Lambda_{\mathrm{h}^{+}} / \Lambda_{\mathrm{e}^{-}}$is not largely affected, being always within the 0.7-1.0 range of values and thus favouring an ambipolar transport (once and again, if all other factors remain the same).

\section{Partial halogenation}

We attempt next to understand if some site-dependent behavior exists, by studying the set of di-substituted molecules shown in Figure 4. These molecules remain planar in all the studied (neutral and charged) states. Actually, upon concerted di-fluorination, reorganization energies remain almost unaf- fected: $\Lambda_{\mathrm{h}^{+}}\left(\Lambda_{\mathrm{e}^{-}}\right)$ranges between 185 (199) and 192 (210) $\mathrm{meV}$ for the 1,2-difluorocoronene and 1,10-difluorocoronene molecules, respectively. The same trend is also observed for $\Lambda_{\mathrm{h}^{+}}\left(\Lambda_{\mathrm{e}^{-}}\right)$upon concerted di-chlorination, with values ranging between 167 and 175 (218 and 222) meV for the 1,2dichlorocoronene and 1,10-dichlorocoronene molecules, respectively. Thus, the ratio $\Lambda_{\mathrm{h}^{+}} / \Lambda_{\mathrm{e}^{--}}$remains close to a value of 0.8 .

It is interesting to note in passing that both $\Lambda_{\mathrm{h}^{+}}$and $\Lambda_{\mathrm{e}^{--}}$ increase with the number of halogen atoms, $N_{X}$. However, the dependence on $N_{X}$ is much weaker for the hole transport than for the electron transport, as can be seen from Figure 5, where values for coronene $\left(N_{X}=0\right)$,

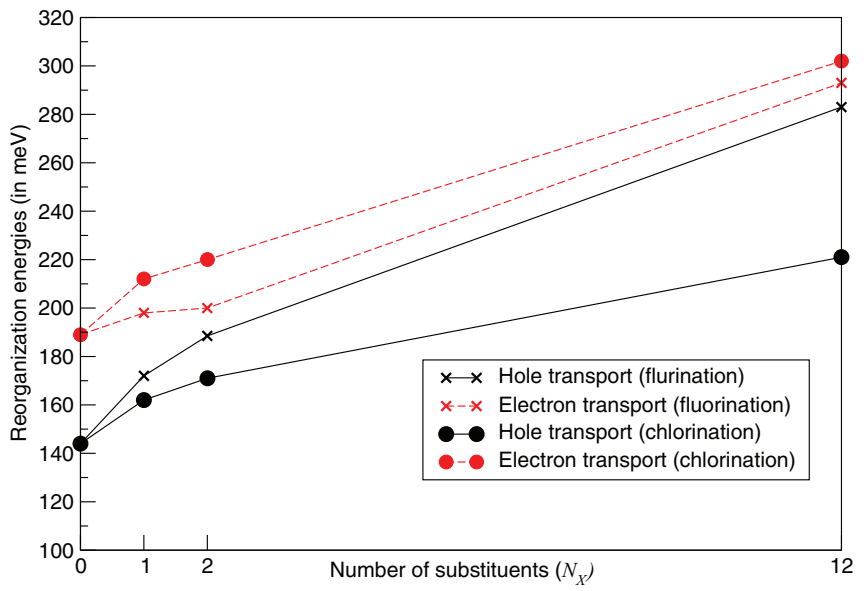

FIG. 5. Evolution of reorganization energies (in meV) values, calculated at the $\mathrm{B}_{\Lambda}$ LYP-D3/def2-TZVP level, for hole and electron transfer in halogenated coronene derivatives as a function of the number $\left(N_{X}\right)$ of halogen atoms. 

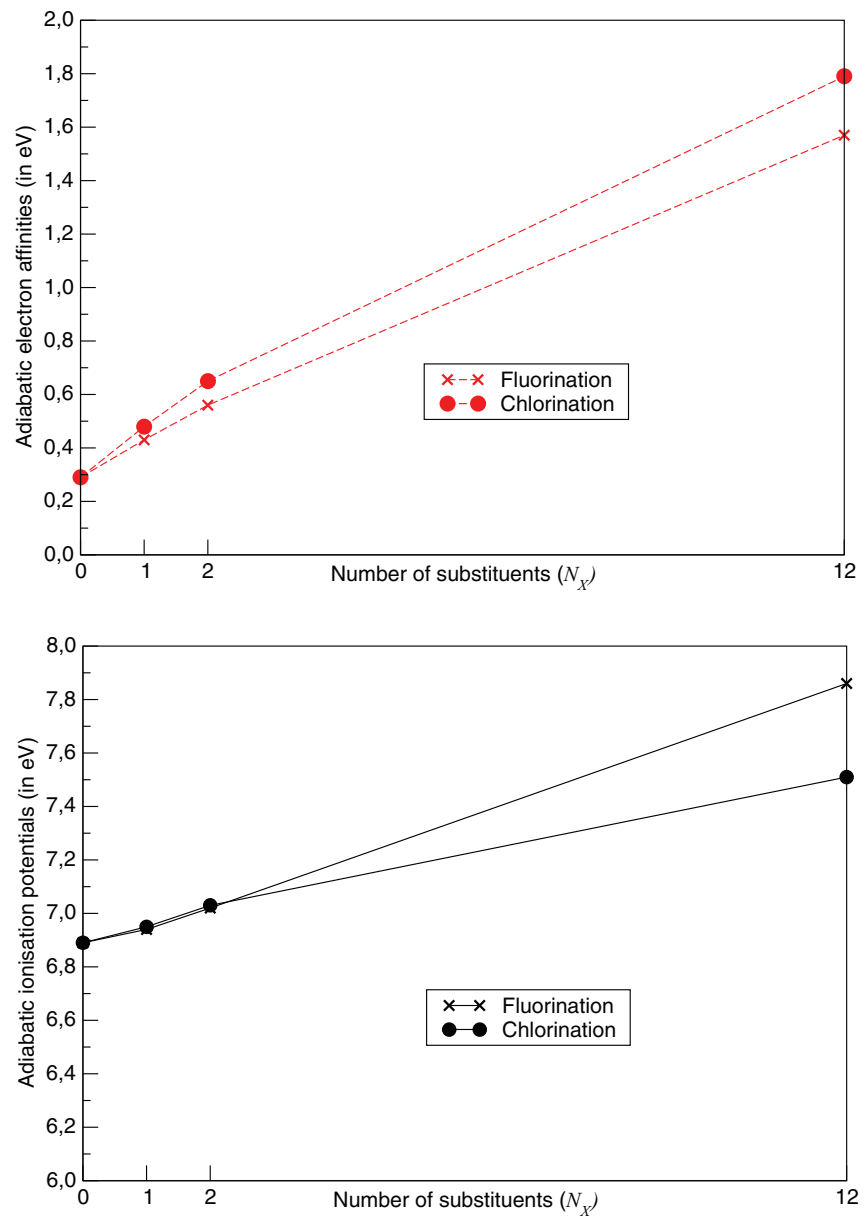

FIG. 6. Evolution of adiabatic ionization potentials (AIP, in eV) and electron affinities (AEA, in eV), calculated at the $\mathrm{B}_{\Lambda}$ LYP-D3/def2-TZVP level, in halogenated coronene derivatives as a function of the number $\left(N_{X}\right)$ of halogen atoms.

mono- $\left(N_{X}=1\right)$, di- $\left(N_{X}=2\right)$, and per-halogenated $\left(N_{X}=12\right)$ molecules are plotted. Note that for di-halogenated derivatives we take the average of values for 1,2- and 1,10-disubstituted molecules. By inspecting the figure, while $\Lambda_{\mathrm{e}^{-}}$follows an almost linear trend for both $\mathrm{F}$ and $\mathrm{Cl}$ derivatives, $\Lambda_{\mathrm{h}^{+}}$roughly depends on $\sqrt{N_{X}}$. The rationale behind the relative trends observed is rooted in the corresponding evolution of the electron affinity and the ionization potential with $N_{X}$, which are listed in Table I, and plotted in Figure 6. Such trends explain why $\Lambda_{\mathrm{h}^{+}}$and $\Lambda_{\mathrm{e}^{-}}$are quite similar to each other for full per-fluorination while for full per-chlorination the former is much larger than the latter. Due to the accumulated features exposed above related to per-chlorination, we will thus mainly concentrate in per-fluorinated derivatives in the following part of the study.

\section{B. Structure and stability of supramolecularly organised samples}

Supramolecular self-assembly of non-planar molecules will increase the intermolecular distance as well as (expectedly) decrease the electronic coupling, even at maximum space-filling arrangements. For instance, dode- cakis(phenylthio)coronene holds an intracolumnar distance between the coronene core of neighboring molecules of around $10 \mathrm{~nm} .{ }^{26}$ On the other hand, the detailed layered structure of samples with large steric hindrance might depend on the nature of the substrate used to grow the thin films, ${ }^{76}$ which further complicates the disclosure of structure-property relationships. Thus, in line with the previous findings, we will concentrate next only on fluorinated coronene molecules due to the interplay between less bulky substituents, having thus preferred planar molecular backbones, and reasonably low reorganization energies for both hole and electron transport. Additionally, the solid-state packing of planar aromatic $\pi$-systems is driven by through-space non-covalent interactions. These molecules would tend to adopt forms keeping their planes parallel to one another, as much as possible, although these weak interactions might also depend on the presence or absence of substituents, either peripheral or within the backbone.

Predicting molecular crystal structures is a very active yet challenging field. ${ }^{77}$ We would like to stress first here that studies on dimer systems cannot fully reflect the complex supramolecular order of crystalline samples, also due to the balance between thermodynamical and kinetic effects. We will analyse apolar, flat-shaped, coronene most stable $\gamma$-type packing first. ${ }^{78-80}$ Also note that optimizations performed on a stacked trimer did not provide further stabilization effects, the energy gained in the aggregation of two monomers to form the dimer was less than $0.2 \mathrm{kcal} \mathrm{mol}^{-1}$ with respect to that obtained for the optimized dimer, also with adjacent molecules staggered along the stacking direction as previously reported. ${ }^{81,82}$ As a matter of example, structures of coronene-type molecules are known to gain additional stabilization from $\mathrm{C} \cdot \mathrm{H}$ interactions between molecules belonging to parallel planes (see Figure 7). Thus, we will concentrate in the following on the most stable arrangement of a pair of coronene molecules, from fully optimized BLYP-D3/def2SVP calculations, which consists in a staggered stacking with the following displacements of the center of mass in the upper molecule with respect to that in the lower one alongside the in-plane $(x, y)$ and perpendicular $(z)$ axes described in Figure 8: $\Delta_{x}=1.37 \AA, \Delta_{y}=0.79 \AA$, and $\Delta_{z}=3.34 \AA$. Note that: (i) the calculated intermolecular distance is close to experimental estimates (3.43-3.46 $\AA$ ); (ii) the lateral displacements also closely agree with other theoretical estimates ${ }^{83,84}$ within $0.05 \AA$; (iii) the stability (association energy) of the dimer, calculated as $E=E_{\text {dimer }}(\Delta d)-2 E_{\text {monomer }}$, being

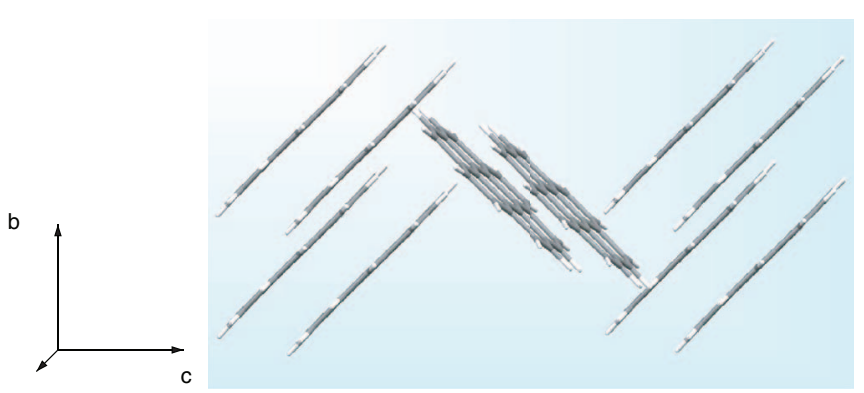

FIG. 7. Unit cell of the coronene crystal structure viewed along the crystal$\operatorname{lographic} b$ axis. 


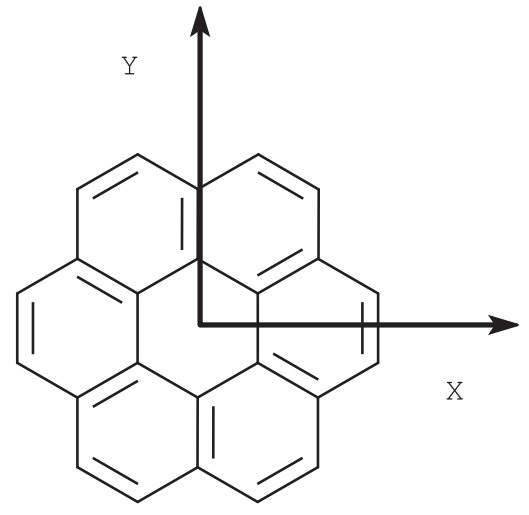

FIG. 8. In-plane $x$ and $y$ directions along which the displacements of the center of mass of the upper monomer are measured with respect to that in the lower one. The $z$ direction is that perpendicular to the $x y$ plane shown. The displacements $\Delta_{x}, \Delta_{y}$, and $\Delta_{z}$ are the projections of the vector joining the two centers of mass along each of the $x, y$, and $z$ directions.

$E_{\text {dimer }}(\Delta d)$ the total energy of the dimer at the optimized intermolecular distance $(\Delta d)$ between the center of mass of the monomers, and with the BLYP-D3/def2-TZVP method, is $-20.80 \mathrm{kcal} \mathrm{mol}^{-1}$; (iv) this stability compares very reasonably with a value obtained here of $-18.71 \mathrm{kcal} \mathrm{mol}^{-1}$ for the $(0, b, 0)$ dimer (central dimer of Figure 7) extracted from the unit cell (the X-ray structure of the coronene crystal was taken from the Crystallography Open Database and processed with the Mercury program ${ }^{85}$ ); (v) the difference in the association energy on going from the cost-effective def2-SVP $\left(-24.52 \mathrm{kcal} \mathrm{mol}^{-1}\right)$ to the sufficiently large def2-TZVP basis set amounts to $3.7 \mathrm{kcal} \mathrm{mol}^{-1}$, which thus forces the use of the latter in the following if one aims at estimating further association energies of nanoaggregates; and (vi) our results consistently agree with the findings recently reported in the literature ${ }^{86-92}$ concerning relative molecular orientation and association energy. Since the crystal, or any other, structure data are not available for supramolecularly organised fluorinated derivatives, to the best of our knowledge, we will rely next on this level of theory (BLYP-D3/def2-TZVP) to analyse the stability of the rest of non-covalently bound molecules.

Given this initial benchmarking, it is both timely and interesting to consider idealised motifs alternating electron-rich (fluorinated derivatives) and electron-deficient (coronene) aromatic cores, to promote face-centered stacking ${ }^{93}$ maximising intramolecular electronic interactions. Note that: (i) a $1: 1$ mixture of benzene and hexafluorobenzene forms a solid composed of layers of alternating molecules, without any experimental indication of partial transfer of charge, which can be thus stabilized by their different (with opposite sign) quadrupole moments; ${ }^{94}$ and (ii) the most stable dimer found in crystals of hexafluorobenzene also exhibits a paralleldisplaced relative orientation. ${ }^{95}$ Table II reports the interaction energies of the $1: 1$ studied complexes (coronene and the fluorinated derivatives included in Figure 4) which, with the exception of coronene : 1,2-difluorocoronene, increase upon fluorination. Furthermore, the intermolecular distance always decreases with respect to the coronene dimer and situates in the 3.0-3.3 $\AA$ range of values. These results perfectly agree with benchmark calculations (the set named as X40) of non-
TABLE II. Estimates at the BLYP-D3/def2-TZVP level of interacting energy $\left(E\right.$, in $\left.\mathrm{kcal} \mathrm{mol}^{-1}\right)$ for a stacked dimer composed of coronene molecule and some of its fluorinated derivatives, as well as the optimized displacement found along the intermolecular distance $\left(\Delta_{z}\right.$, in $\AA$ ).

\begin{tabular}{lcc}
\hline \hline Coronene derivative & $E$ & $\Delta_{z}$ \\
\hline Coronene : coronene & -20.80 & 3.34 \\
Coronene : 1-fluorocoronene & -20.93 & 3.35 \\
Coronene : 1,2-difluorocoronene & -20.09 & 3.32 \\
Coronene : 1,3-difluorocoronene & -22.12 & 3.29 \\
Coronene : 1,4-difluorocoronene & -21.71 & 3.32 \\
Coronene : 1,5-difluorocoronene & -21.33 & 3.33 \\
Coronene : 1,6-difluorocoronene & -21.54 & 3.33 \\
Coronene : 1,7-difluorocoronene & -22.11 & 3.31 \\
Coronene : 1,9-difluorocoronene & -21.55 & 3.33 \\
Coronene : 1,10-difluorocoronene & -21.87 & 3.32 \\
Coronene : per-fluorocoronene & -28.90 & 3.25 \\
\hline \hline
\end{tabular}

covalent interactions of smaller halogenated molecules ${ }^{96}$ and with a recent study on substituted coronene molecules although in a rigid-monomer (non-optimized dimer systems) fashion. ${ }^{97}$ As it should be, the dimer stabilized the most is coronene : per-fluorocoronene, also showing almost eclipsed hydrogen/fluorine atoms, see Figure 9, which may be thus a suitable engineered molecule for further studies. Also note that an envisioned per-fluorocoronene : per-fluorocoronene crystal might prefer a larger parallel-displaced or even edgeto-face stacking motif in the solid-state, since both interacting molecules will be now electron-rich aromatics. Actually, the interaction energy calculated for such a slipped cofacial dimer $\left(-27.08 \mathrm{kcal} \mathrm{mol}^{-1}\right)$ is similar to that for the corresponding coronene : per-fluorocoronene, $-28.90 \mathrm{kcal} \mathrm{mol}^{-1}$ as reported in Table II, which seems to be large enough; thus, this molecule will also be chosen as a target to also estimate its charge mobility value compared to the reference coronene : coronene system.

\section{Estimating charge mobility values}

\section{Electronic couplings}

We present first (Table III) the results of calculated electronic couplings at previously optimized geometries for the corresponding dimers. In the case of coronene, these values are larger for hole than for electron transport (206 vs $101 \mathrm{meV}$, respectively), close to previously reported results of 167 and $65 \mathrm{meV}$ obtained in Ref. 72 with the PBE/TZVP method and at the $\omega \mathrm{B} 97 \mathrm{XD}^{98}$ optimized geometry of the dimer. We have also checked the results for electronic couplings at the corresponding crystal geometry of the dimer: our values for hole and for electron transport (161 vs 86 $\mathrm{meV}$, respectively) still hold the trend found before. Furthermore, when one uses an approximate method for calculating the electronic coupling values in the coronene case, like the "energy splitting in dimer" approach where $V_{\text {if }}$ is taken as half the splitting of the LUMO (HOMO) levels in a neutral dimer for electron (hole) transport, we obtain the same trend (even if values are strongly underestimated) no matter if RHF, INDO/S, or DFT-based results are listed: 83 and $11 \mathrm{meV}$ 

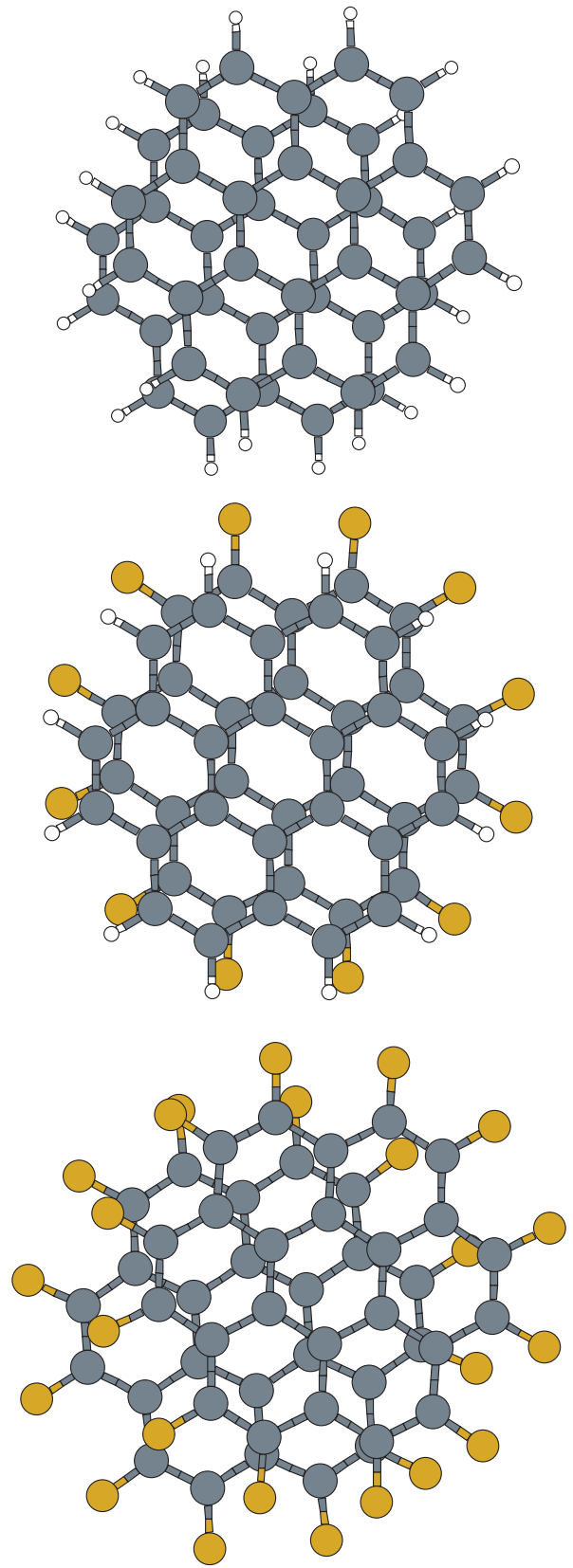

FIG. 9. Optimized structure of a dimer of (from top to bottom) coronene : coronene, coronene : per-fluorocoronene, and per-fluorocoronene : perfluorocoronene within a stack from perpendicular view, calculated at the BLYP-D3/def2-SVP level.

(RHF/cc-pVDZ) or 68 and $16 \mathrm{meV}$ (INDO/S) or 53 and 14 $\mathrm{meV}$ (BLYP/cc-pVDZ) for hole or electron transport, respectively, all of them at the BLYP-D3/def2-SVP optimized geometry of the dimer. All-in-all, we firmly rely on our cal-

TABLE III. Estimates at the UHF/cc-pVDZ level of hole and electron electronic couplings $\left(\mathrm{V}_{\mathrm{if}}\right.$, in meV) and corresponding charge transfer rates $\left(\mathrm{k}_{\mathrm{CT}}\right.$, in $\times 10^{13} \mathrm{~s}^{-1}$ ) for the optimized structures of the indicated dimers.

\begin{tabular}{lcccc}
\hline \hline Molecule & $\mathrm{V}_{\mathrm{if}}\left(\mathrm{h}^{+}\right)$ & $\mathrm{k}_{\mathrm{CT}}\left(\mathrm{h}^{\cdot+}\right)$ & $\mathrm{V}_{\mathrm{if}}\left(\mathrm{e}^{\cdot-}\right)$ & $\mathrm{k}_{\mathrm{CT}}\left(\mathrm{e}^{\cdot-}\right)$ \\
\hline Coronene & 206 & 24.9 & 101 & 3.51 \\
Per-fluorocoronene & 116 & 1.53 & 22 & 0.05 \\
\hline \hline
\end{tabular}

culated UHF/cc-pVDZ//BLYP-D3/def2-SVP electronic coupling values to estimate next charge transfer rates.

\section{Hole and electron transfer rates}

It remains important to remark that a quasi-columnar packing is known to highly correlate with large electronic coupling values, ${ }^{99,100}$ and thus that the previously afforded systems (coronene and per-fluorocoronene) might behave adequately upon charging mainly because of the large stability of the corresponding per-fluorocoronene dimer, which would predict its existence in supramolecularly ordered samples. We will limit ourselves in the following to the study of these two systems mainly on a relative basis. We note that the electronic coupling between other crystallographic directions (parallel stacks) can be considered small, ${ }^{101,102}$ although might however promote large anisotropy of charge carrier mobilities.

First, before feeding the results of our calculations into Eq. (2) we also need to address the reorganization energy of the dielectric medium upon arrival of the charge carrier. This contribution (dubbed $\Lambda_{s}$ or external reorganization energy) adds to the values of $\Lambda$ given by Eq. (3), being now $\Lambda^{\prime}=\Lambda+\Lambda_{s}$, and it is conveniently represented by a tunable parameter ${ }^{103,104}$ due to existing difficulties to its direct theoretical estimates. ${ }^{105}$ Besides this, it has also been documented before for oligoacenes how small its value is (e.g., for anthracene single crystals a value of only $52 \mathrm{meV}$ was predicted $\left.{ }^{106}\right)$ as well as its progressive decrease with system size. ${ }^{107}$ Therefore, we will use the following values, to be considered as an upper limit and based on the fact that this contribution is smaller the less polar the medium is, for coronene $(50 \mathrm{meV})$ and per-fluorocoronene $(75 \mathrm{meV})$, the latter being higher due to (possible) deformations of the lattice in the immediate vicinity of the polarised atoms.

Following these steps, we obtain a hopping rate for hole transport of $2.5 \times 10^{14} \mathrm{~s}^{-1}$ for coronene, see Table III. This value is comparable to theoretical estimates made for best suited materials currently used, such as rubrene $\mathrm{e}^{108}$ or phtalocyanines. ${ }^{109}$ When looking at corresponding rates for electron transport, we obtain a value of $3.5 \times 10^{13} \mathrm{~s}^{-1}$, which also compares favourably with halogenated oligoacenes ${ }^{74}$ although it is still an order of magnitude lower than for other materials such as some state-ofthe-art pentacenequinones. ${ }^{110}$ Most importantly, these rates might anticipate a great behavior, because for purely diffusive quasi-1D motion, the Einstein-Smoluchowski equation reads as $^{30,111}$

$$
\mu=\frac{(\Delta d)^{2} \mathrm{k}^{\mathrm{CT}}}{k_{B} \Theta},
$$

with $\Delta d$ the distance between the center of mass of both molecules $(3.70 \AA)$ as they situate in the interacting optimized dimer. This expression leads to hole (electron) mobilities of roughly $13 \mathrm{~cm}^{2} \mathrm{~V}^{-1} \mathrm{~s}^{-1}\left(1.9 \mathrm{~cm}^{2} \mathrm{~V}^{-1} \mathrm{~s}^{-1}\right)$ and a relative $\mu\left(\mathrm{h}^{+}\right) / \mu\left(\mathrm{e}^{\cdot-}\right) \simeq 7$.1. Inspecting now the results obtained for the per-fluorocoronene system, we obtain smaller hopping rates than for coronene due to an increase (decrease) of 
reorganization energies (electronic couplings): see Table III. We will show next the calculated mobility values of this derivative as compared to those obtained for the coronene case, rather than trying to provide fully quantitative estimates of charge mobility values, by relying on the expression

$$
\frac{\mu_{\text {derivative }}}{\mu_{\text {coronene }}} \propto \frac{\mathrm{V}_{\text {derivative }}^{2}}{\mathrm{~V}_{\text {coronene }}^{2}} \sqrt{\frac{\Lambda_{\text {coronene }}}{\Lambda_{\text {derivative }}}} e^{\left(\Lambda_{\text {coronene }}-\Lambda_{\text {derivative }}\right) / 4 k_{B} T},
$$

with $\mathrm{V}$ and $\Lambda$ the above calculated values, and which drops a ratio of $0.06(0.01)$ between the expected hole (electron) mobilities of per-fluorocoronene derivative and coronene.

\section{CONCLUSIONS}

We would like to emphasise that the various approaches followed in the literature may vary in many regards, including the theoretical method(s) employed to estimate the key magnitudes entering into the formulae, but can be truly complementary and may result in further advances within the field. Note that we use a model where the charges interact strongly with intermolecular vibrations, using thus a localized picture and corresponding transfer rates to estimate the transport, according to the magnitude of electron-phonon interactions: $V_{\text {if }}$ is always found smaller than $\Lambda$ for the molecules under investigation. In this regards, let us now summarize the main findings obtained in this work.

- We notice that the induced electroactive effects upon per-halogenation of coronene, together with further structural "twisting" by steric hindrance caused in the case of per-chlorination, favours the charge localization, causing an increase of both the IP and the EA, which in the latter case may facilitate the electron injection from the metallic electrode.

- However, these increases also lead to larger reorganization energies. Indeed, we have observed a correlation between the $\Lambda_{\mathrm{h}^{+}}\left(\Lambda_{\mathrm{e}^{--}}\right)$values and the IP (EA) as $N_{X}$ increases, being $N_{X}$ the number of halogen atoms, which leads to almost equal values for hole and electron reorganization energies in the case of perfluorination. On the other hand, the calculations performed on several di-fluorinated derivatives show that the ratio $\Lambda_{\mathrm{h}^{+}} / \Lambda_{\mathrm{e}^{--}}$remains almost constant irrespective of the relative position between the fluorine atoms.

- The geometry optimizations performed on isolated coronene dimers indicate that calculations using stateof-the-art dispersion-corrected functionals, such as BLYP-D3, are accurate enough to yield reasonable estimates of association energies and intermolecular distances, provided that a sufficiently large basis, like the def2-TZVP one, is used.

- The optimizations done on mixed dimers including one coronene molecule and one per-fluorinated derivative indicate that per-fluorination has shorter internuclear distances than partially fluorinated ones, be- ing also much more most stable, with an association energy similar to that calculated for two fully perfluorinated coronenes. This is in agreement with recent studies on benzene-hexafluorobenzene complexes, and points towards these 1:1 mixed aggregates as interesting materials for subsequent studies.

- The electronic couplings calculated for hole and electron transport of coronene dimers point towards a not-so-strong ambipolar behavior, with a quotient $\mu_{\mathrm{h}^{+}} / \mu_{\mathrm{e}^{-}}$close to seven, with $\mu$ estimated via Eqs. (2) and (6). As for per-fluorocoronene, the electronic coupling for hole transport is halved with respect to that of coronene, but that for electron transport is five times lower. This, combined with larger reorganization energies, leads to hole (electron) mobilities that are around $6 \%(1 \%)$ of those estimated for coronene.

\section{ACKNOWLEDGMENTS}

This work is supported by the "Ministerio de Educación y Ciencia" of Spain and the "European Regional Development Fund" through Project No. CTQ2011-27253.

${ }^{1}$ V. Coropceanu, J. Cornil, D. A. da Silva Filho, Y. Olivier, R. Silbey, and J.-L. Bredás, Chem. Rev. 107, 926 (2007).

${ }^{2}$ V. Stehr, J. Pfister, R. F. Fink, B. Engels, and C. Deibel, Phys. Rev. B 83, 155208 (2011).

${ }^{3}$ J. Cornil, J.-L. Brédas, J. Zaumseil, and H. Sirringhaus, Adv. Mater. 19, 1791 (2007).

${ }^{4}$ A. L. Appleton, S. M. Brombosz, S. Barlow, J. S. Sears, J.-L. Brédas, S. R. Marder, and U. H.-F. Bunz, Nat. Commun. 1, 91 (2010).

${ }^{5}$ M. Stolar and T. Baumgartner, Phys. Chem. Chem. Phys. 15, 9007 (2013).

${ }^{6}$ X. Gao and Y. Hu, J. Mater. Chem. C 2, 3099 (2014).

${ }^{7}$ X. Zhan, A. Facchetti, S. Barlow, T. J. Marks, M. A. Ratner, M. R. Wasielewski, and S. R. Marder, Adv. Mater. 23, 268 (2011).

${ }^{8}$ Q. Meng and W. Hu, Phys. Chem. Chem. Phys. 14, 14152 (2012).

${ }^{9}$ J. Mei, Y. Diao, A. L. Appleton, L. Fand, and Z. Bao, J. Am. Chem. Soc. 135, 6724 (2013).

${ }^{10}$ B. Zhang, Y.-H. Kan, Y. Geng, Y.-A. Duan, H.-B. Li, J. Hua, and Z.-M. Su, Org. Electron. 14, 1359 (2013).

${ }^{11}$ M. Kitamura and Y. Arakawa, J. Phys.: Condens. Matter 20, 184011 (2008).

${ }^{12}$ C. Wang, H. Dong, W. Hu, Y. Li, and D. Zhu, Chem. Rev. 112, 2208 (2012).

${ }^{13}$ Y. Yamaguchi, K. Ogawa, K.-I. Nakayama, Y. Ohba, and H. Katagiri, J. Am. Chem. Soc. 135, 19095 (2013).

${ }^{14}$ X. Feng, V. Marcon, W. Pisula, M. R. Hansen, J. Kirkpatrick, F. C. Grozema, D. Andrienko, K. Kremer, and K. Müllen, Nat. Mater. 8, 421 (2009).

${ }^{15}$ I. Díez-Pérez, Z. Li, J. Hihath, J. Li, C. Zhang, X. Yang, L. Zang, Y. Dai, X. Feng, K. Müllen, and N. Tao, Nat. Commun. 1, 31 (2010).

${ }^{16}$ A. A. Sagade, K. V. Rao, U. Mogera, S. J. George, A. Datta, and G. U. Kulkarni, Adv. Mater. 25, 559 (2013).

${ }^{17}$ M. Mas-Torrent and C. Rovira, Chem. Rev. 111, 4833 (2011).

${ }^{18}$ A. M. van de Craats, N. Stutzmann, O. Bunk, M. M. Nielsen, M. Watson, K. Müllen, H. D. Chanzy, H. Sirringhaus, and R. H. Friend, Adv. Mater. 15, 495 (2003).

${ }^{19}$ W. Pisula, A. Menon, M. Stepputat, I. Lieberwirth, U. Kolb, A. Tracz, H. Sirringhaus, T. Pakula, and K. Müllen, Adv. Mater. 17, 684 (2005).

${ }^{20}$ S. E. Wheeler, J. Am. Chem. Soc. 133, 10262 (2011).

${ }^{21}$ W. Wang, Y. Zhang, and Y.-B. Wang, J. Chem. Phys. 140, 094302 (2014).

${ }^{22}$ B. Milián-Medina, D. Beljonne, H.-J. Egelhaaf, and J. Gierschner, J. Chem. Phys. 126, 111101 (2007).

${ }^{23}$ Y. Sakamoto, T. Suzuki, M. Kobayashi, Y. Gao, Y. Fukai, Y. Inoue, F. Sato, and S. Tokito, J. Am. Chem. Soc. 126, 8138 (2004).

${ }^{24}$ J. E. Anthony, Chem. Rev. 106, 5028 (2006). 
${ }^{25}$ X. Wan, K. Chen, J. Du, D. Liu, J. Chen, X. Lai, W. Xie, and J. Xu, J. Phys. Chem. C 117, 4800 (2013).

${ }^{26}$ P. Kowalzik, S. Rathgeber, S. Karthauser, R. Waser, N. Schnaebele, J.-M. Raimundo, and M. Gingras, New J. Chem. 36, 477 (2012).

${ }^{27}$ T. Maeda, H. Fujimoto, M. Yoshikawa, M. Saito, Y. Okita, K. Onoe, and Y. Arai, Japanese patent JP05194972 (August 3, 1993).

${ }^{28}$ D. P. McMahon and A. Troisi, Phys. Chem. Chem. Phys. 13, 10241 (2011).

${ }^{29}$ J.-L. Brédas, D. Beljonne, V. Coropceanu, and J. Cornil, Chem. Rev. 104, 4971 (2004).

${ }^{30}$ F. C. Grozema and L. D. A. Siebbeles, Int. Rev. Phys. Chem. 27, 87 (2008).

${ }^{31}$ D. Cheung and A. Troisi, Phys. Chem. Chem. Phys. 10, 5941 (2008).

${ }^{32}$ S. Stafström, Chem. Soc. Rev. 39, 2484 (2010).

${ }^{33}$ R. A. Marcus, Rev. Mod. Phys. 65, 599 (1993).

${ }^{34}$ J. Bisquert and R. A. Marcus, Top. Curr. Chem. 352, 325-395 (2014).

${ }^{35}$ N. G. Martinelli, Y. Olivier, S. Athanasopoulos, M.-C. Ruiz-Delgado, K. R. Pigg, D. A. da Silva Filho, R. S. Sánchez-Cabrera, E. Venuti, R. G. Della Valle, J.-L. Brédas, D. Beljonne, and J. Cornil, ChemPhysChem 10, 2265 (2009).

${ }^{36}$ D. P. McMahon and A. Troisi, ChemPhysChem 11, 2067 (2010).

${ }^{37}$ L. Wang, G. Nan, X. Yang, Q. Peng, Q. Li, and Z. Shuai, Chem. Soc. Rev. 39, 423 (2010).

${ }^{38}$ A. Troisi, Adv. Polym. Sci. 223, 259 (2010).

${ }^{39}$ A. Troisi, Chem. Soc. Rev. 40, 2347 (2011).

${ }^{40}$ Z. Shuai, L. Wang, and Q. Li, Adv. Mater. 23, 1145 (2011).

${ }^{41}$ F. Neese, WIREs Comput. Mol. Sci. 2, 73 (2012).

${ }^{42}$ F. Neese, F. Wennmohs, A. Hansen, and U. Becker, Chem. Phys. 356, 98 (2009).

${ }^{43}$ K. Eichkorn, F. Weigend, O. Trutler, and R. Ahlrichs, Theor. Chem. Acc. 97, 119 (1997)

${ }^{44}$ M. Valiev, E. J. Bylaska, N. Govind, K. Kowalski, T. P. Straatsma, H. J. J. van Dam, D. Wang, J. Nieplocha, E. Apra, T. L. Windus, and W. A. de Jong, Comput. Phys. Commun. 181, 1477 (2010).

${ }^{45}$ A. Farazdel, M. Dupuis, E. Clementi, and A. Aviram, J. Am. Chem. Soc. 112, 4206 (1990).

${ }^{46}$ K. Rosso, D. M. A. Smith, and M. Dupuis, J. Chem. Phys. 118, 6455 (2003).

${ }^{47}$ P. Liao, M. C. Toroker, and E. A. Carter, Nano Lett. 11, 1775 (2011).

${ }^{48}$ M. Pavanello, T. Van Voorhis, L. Visscher, and J. Neugebauer, J. Chem. Phys. 138, 054101 (2013).

${ }^{49}$ R. S. Sánchez-Carrera, V. Coropceanu, D. A. da Silva, R. Friedlein, W. Osikowicz, R. Murdey, C. Suess, W. R. Salaneck, and J.-L. Brédas, J. Phys. Chem. B 110, 18904 (2006).

${ }^{50}$ C. Sutton, J. S. Sears, V. Coropceanu, and J.-L. Brédas, J. Phys. Chem. Lett. 4, 919 (2013).

${ }^{51}$ V. Coropceanu, J. M. André, M. Malagoli, and J.-L. Brédas, Theor. Chem. Acc. 110, 59 (2003).

${ }^{52}$ J. C. Sancho-García, Chem. Phys. 331, 321 (2007).

${ }^{53}$ M. Malagoli, V. Coropceanu, D. A. da Silva Filho, and J.-L. Brédas, J. Chem. Phys. 120, 7490 (2004).

${ }^{54}$ A. D. Becke, Phys. Rev. A 38, 3098 (1988).

${ }^{55}$ C. Lee, W. Yang, and R. G. Parr, Phys. Rev. B 37, 785 (1988).

${ }^{56}$ F. Weigend and R. Ahlrichs, Phys. Chem. Chem. Phys. 7, 3297 (2005).

${ }^{57}$ S. Grimme, J. Antony, S. Ehrlich, and H. Krieg, J. Chem. Phys. 132, 154104 (2010).

${ }^{58}$ S. Grimme, S. Ehrlich, and L. Goerigk, J. Comput. Chem. 32, 1456 (2011).

${ }^{59}$ O. A. von Lilienfeld and A. Tkatchenko, J. Chem. Phys. 132, 234109 (2010).

${ }^{60}$ R. Podeszwa, B. M. Rice, and K. Szalewicz, Phys. Rev. Lett. 101, 115503 (2008).

${ }^{61}$ W. Reckien, F. Janetzko, M. F. Peintinger, and T. Bredow, J. Comput. Chem. 33, 2023 (2012).

${ }^{62}$ M. D. Newton, Chem. Rev. 91, 767 (1991).

${ }^{63}$ J. Huang and M. Kertesz, Chem. Phys. Lett. 390, 110 (2004).

${ }^{64}$ A. Migliore, J. Chem. Theory Comput. 7, 1712 (2011).

${ }^{65}$ M. Ottonelli, M. Piccardo, D. Duce, S. Thea, and G. Dellepiane, Energy Proc. 31, 31 (2012).

${ }^{66}$ S. Erkoç, F. Erkoç, and L. Türker, J. Mol. Struct.: THEOCHEM 535, 159 (2001).

${ }^{67}$ X. Chi, D. Li, H. Zhang, Y. Chen, V. García, C. García, and T. Siegrist, Org. Electron. 9, 234 (2008).

${ }^{68}$ R. Cardia, G. Malloci, A. Mattoni, and G. Capellini, J. Phys. Chem. A 118, 5170 (2014).
${ }^{69}$ M. C. Rúuiz-Delgado, K. R. Pigg, D. A. da Silva Filho, N. E. Gruhn, Y. Sakamoto, T. Suzuki, R. Malavé-Osuna, J. Casado, V. Hernández, J. T. López-Navarrete, N. G. Martinelli, J. Cornil, R. S. Sánchez-Carrera, V. Coropceanu, and J.-L. Brédas, J. Am. Chem. Soc. 131, 1502 (2009).

${ }^{70}$ D. Schröder, J. Loos, H. Schwarz, R. Thissen, D. V. Preda, L. T. Scott, D. Caraiman, M. V. Frach, and D. K. Böhme, Helv. Chim. Acta 84, 1625 (2001).

${ }^{71}$ M. A. Duncan, A. M. Knight, Y. Negishi, S. Nagao, Y. Nakamura, A. Kato, A. Nakajima, and K. Kaya, Chem. Phys. Lett. 309, 49 (1999).

${ }^{72}$ S. Sanyal, A. K. Manna, and S. K. Pati, J. Phys. Chem. C 117, 825 (2013).

${ }^{73}$ J. C. Sancho-García and A. J. Pérez-Jiménez, J. Chem. Phys. 131, 084108 (2009).

${ }^{74}$ J. C. Sancho-García, A. J. Pérez-Jiménez, Y. Olivier, and J. Cornil, Phys. Chem. Chem. Phys. 12, 9381 (2010).

${ }^{75}$ D. A. da Silva Filho, V. Coropceanu, N. E. Gruhn, P. H. de Oliveira Neto, and J.-L. Brédas, Chem. Commun. 49, 6069 (2013).

${ }^{76}$ S. C. B. Mannsfeld, A. Virkar, C. Reese, M. F. Toney, and Z. Bao, Adv. Mater. 21, 2294 (2009).

${ }^{77}$ G. J. O. Beran, S. Wen, K. Nanda, Y. Huang, and Y. Heit, Top. Curr. Chem. 345, 59-93 (2014).

${ }^{78}$ G. R. Desiraju and A. Gavezzotti, J. Chem. Soc., Chem. Commun. 1989, 621.

${ }^{79}$ G. R. Desiraju and A. Gavezzotti, Acta Crystallogr. B45, 473 (1989).

${ }^{80}$ A. H. Matsui and K.-I. Mizuno, J. Phys. D: Appl. Phys. 26, B242 (1993).

${ }^{81}$ M. Rapacioli, F. Calvo, F. Spiegelman, C. Joblin, and D. J. Wales, J. Phys. Chem. A 109, 2487 (2005).

${ }^{82}$ L. M. Da Costa, S. R. Stoyanov, S. Gusarov, P. R. Seidl, J. W. de M. Carneiro, and A. Kovalenko, J. Phys. Chem. A 118, 896 (2014).

${ }^{83}$ T. Janowski, R. A. Ford, and P. Pulay, Mol. Phys. 108, 249 (2010).

${ }^{84}$ R. Podeszwa, J. Chem. Phys. 132, 044704 (2010).

${ }^{85}$ C. F. Macrae, I. J. Bruno, J. A. Chisholm, P. R. Edgington, P. McCabe, E. Pidcock, L. Rodríguez-Monge, R. Taylor, J. van de Streek, and P. A. Wood, J. Appl. Crystallogr. 41, 466 (2008).

${ }^{86}$ S. Grimme, C. Mück-Lichtenfeld, and J. Antony, J. Phys. Chem. C 111, 11199 (2007)

${ }^{87}$ O. I. Obolensky, V. V. Semenikhina, A. V. Solov'yov, and W. Greiner, Int. J. Quantum Chem. 107, 1336 (2007).

${ }^{88}$ Y. Zhao and D. G. Truhlar, J. Phys. Chem. C 112, 4061 (2008).

${ }^{89}$ I. D. Mackie and G. A. DiLabio, J. Phys. Chem. A 112, 10968 (2008).

${ }^{90}$ C. Feng, C. S. Lin, W. Fan, R. Q. Zhang, and M. A. Van Hove, J. Chem. Phys. 131, 194702 (2009).

${ }^{91}$ T. Janowski and P. Pulay, J. Am. Chem. Soc. 134, 17520 (2012).

${ }^{92}$ J. Li, Y. Liu, Y. Qian, L. Li, L. Xie, J. Shang, T. Yu, M. Yi, and W. Huang, Phys. Chem. Chem. Phys. 15, 12694 (2013).

${ }^{93}$ C. R. Martínez and B. L. Iverson, Chem. Sci. 3, 2191 (2012).

${ }^{94}$ J. H. Williams, Acc. Chem. Res. 26, 593 (1993).

${ }^{95}$ J. Trnka, R. Sedlak, M. Koláŕ, and P. Hobza, J. Phys. Chem. A 117, 4331 (2013).

${ }^{96}$ J. Řezàč, K. E. Riley, and P. Hobza, J. Chem. Theory Comput. 8, 4285 (2012).

${ }^{97}$ S. E. Wheeler, CrystEngComm 14, 6140 (2012).

${ }^{98}$ J.-D. Chai and M. Head-Gordon, Phys. Chem. Chem. Phys. 10, 6615 (2008).

${ }^{99}$ V. Lemaur, D. A. da Silva Filho, V. Coropceanu, M. Lehmann, Y. Geerts, J. Piris, M. G. Debije, A. M. van de Craats, K. Senthilkumar, L. D. A. Siebbeles, J. M. Warman, J.-L. Brédas, and J. Cornil, J. Am. Chem. Soc. 126, 3271 (2004).

${ }^{100}$ Y. Olivier, L. Muccioli, V. Lemaur, Y. H. Geerts, C. Zannoni, and J. Cornil, J. Phys. Chem. B 113, 14102 (2009).

${ }^{101}$ L. Viani, Y. Olivier, S. Athanasopoulos, D. A. da Silva Filho, J. Hulliger, J.-L. Brédas, J. Gierschner, and J. Cornil, ChemPhysChem 11, 1062 (2010).

${ }^{102}$ G. Yang, Y. Si, Y. Geng, F. Yu, Q. Wu, and S. Su, Theor. Chem. Acc. 128, 257 (2011).

${ }^{103}$ V. Lemaur, M. Steel, D. Beljonne, J.-L. Brédas, and J. Cornil, J. Am. Chem. Soc. 127, 6077 (2005).

${ }^{104}$ S. F. Nelsen and J. R. Pladziewicz, in Rate Constant Calculations for Thermal Reactions: Methods and Applications, edited by H. Dacosta and M. Fan (John Wiley and Sons, 2012).

${ }^{105}$ J. E. Norton and J.-L. Brédas, J. Chem. Phys. 128, 034701 (2008).

${ }^{106}$ N. G. Martinelli, J. Idé, R. S. Sánchez-Carrera, V. Coropceanu, J.-L. Brédas, L. Ducasse, F. Castet, J. Cornil, and D. Beljonne, J. Phys. Chem. C 114, 20678 (2010). 
${ }^{107}$ D. P. McMahon and A. Troisi, J. Phys. Chem. Lett. 1, 941 (2010).

${ }^{108}$ D. A. da Silva Filho, E.-G. Kim, and J.-L. Bredás, Adv. Mater. 17, 1072 (2005).

${ }^{109}$ L. Li, Q. Tang, H. Li, X. Yang, W. Hu, Y. Song, Z. Shuai, W. Xu, Y. Liu, and D. Zhu, Adv. Mater. 19, 2613 (2007).
${ }^{110}$ J. C. Sancho-García, J. Chem. Phys. 136, 174703 (2012).

${ }^{111}$ C. Gollub, S. Avdoshenko, R. Gutierrez, Y. Berlin, and G. Cuniberti, Isr. J. Chem. 52, 452 (2012).

${ }^{112}$ See supplementary material at http://dx.doi.org/10.1063/1.4897205 for more theoretical details. 